\title{
A Balls-and-Bins Model of Trade: Reply
}

\author{
By Roc Armenter And Miklós Koren*
}

\begin{abstract}
Blum, Claro, and Horstmann (2016) make two statements about the balls-and-bins model of Armenter and Koren (2014). First, that using firm-level shipment data changes some of our results. Second, that the balls-and-bins model is not an appropriate statistical method. We respond to the first statement and argue that the second statement is unfounded and unrelated to the first. Indeed, the work of Blum, Claro, and Horstmann (2016) is a perfect example of how to use balls-and-bins in a rich dataset to spot interesting data patterns. (JEL F11, F14)
\end{abstract}

Blum, Claro, and Horstmann (2016) - henceforth, BCH-report the fraction of exporters selling to single destinations and/or a single product, as well as their share in total export revenues, using firm-level data on Chilean exports. To note one example, around half of exporters only ship to one country. These single-country exporters account for 2.9 percent of all Chilean non-copper exports in 2006.

$\mathrm{BCH}$ then contrast these data moments against those predicted by the "balls-and-bins model" of Armenter and Koren (2014)-henceforth, AK. In this model, each shipment is randomly assigned a destination country and a product category, with a probability given for each country and each product. The balls-and-bins model generates about half of the single-destination and single-product exporters observed in the data. Single-destination-single-product exporters are also underpredicted by balls-and-bins, although not by as much.

This stands in contrast with what we found for the United States in AK, where the balls-and-bins predictions on firm-level export patterns lined up closely with the data. 1 As argued by $\mathrm{BCH}$, the likely culprit is the imputation method used in AK to circumvent the lack of data on firm-level shipments. We assigned each exporter a number of shipments - that is, balls - by dividing the firm's revenue by the average shipment size and rounding up to the nearest integer. $\mathrm{BCH}$ are able to observe the actual number of firm-level shipments and report that the imputation in $\mathrm{AK}$

\footnotetext{
* Armenter: Federal Reserve Bank of Philadelphia, 10 Independence Mall, Philadelphia, PA 19106 (e-mail: roc.armenter@phil.frb.org); Koren: Central European University, Nador utca 9., Budapest 1051, Hungary, MTA, KRTK, and CEPR (e-mail: korenm@ceu.edu). Koren acknowledges financial support from the European Research Council under the European Union's Seventh Framework Program (FP7/2007-2013) ERC grant agreement number 313164. The authors declare that they have no relevant or material financial interests that relate to the research described in this paper. The views expressed here do not necessarily reflect the views of the Federal Reserve Bank of Philadelphia or the Federal Reserve System.

Go to http://dx.doi.org/10.1257/aer.20151233 to visit the article page for additional materials and author disclosure statement $(\mathrm{s})$.

${ }^{1}$ The model's predictions for the frequency of product-country-level zeros remain as closely aligned with the Chilean data as they are for the United States.
} 
overestimates the number of single-shipment exporters. This, in turn, increases the number of single-product and single-destination exporters predicted by the balls-and-bins model.

In absence of direct firm-level measures of shipments, we have explored various calibrations for the size of shipments and their distribution across firms in online Appendix B of AK. We varied the size of shipments between $\$ 2,500$ and $\$ 500,000$, a factor of 200. Relative to this enormous variation in shipment size, some of the predicted statistics about the extensive margin changed little. For example, the share of firm-country zeros in trade only varied between 86 and 99 percent (online Appendix Tables 2 and 3 of AK). Some statistics changed more. The fraction of single-country exporters varied between 15 and 77 percent.

We unfortunately remain unable to access US firm-level shipment data, so we cannot check whether the relationship between firm and shipment size also holds there. Given the results in $\mathrm{BCH}$, we suspect that our imputation method indeed overstated the number of single-shipment firms. In doing so, we likely underestimated the amount of information in the share of single-destination and single-product exporters.

We commend the simultaneous use of firm- and shipment-level data by $\mathrm{BCH}$. Theirs is an ideal dataset to study the extensive margin of countries and products in trade. It identifies decision-making units (firms), and it reports actual units of observation (shipments). As AK make clear, the ideal procedure is to use the actual number of shipments. Using this procedure, $\mathrm{BCH}$ conclude that single-country and single-product exporters are overrepresented in Chile relative to the balls-and-bins benchmark. It seems that Chilean exporters_-particularly small ones-find it hard to reach multiple export destinations. A structural model matching these statistics will help us understand better the barriers to trade. ${ }^{2}$

$\mathrm{BCH}$, however, do not make use of their rich data to develop such a model. Instead, they argue that the fact that firms differ in the size of their shipments invalidates the balls-and-bins methodology.

We believe that their argument is a fallacy by association, indicting the whole methodology on the basis of an imperfect data imputation. As we explained in AK, we used the assumption of constant shipment size solely due to a data limitation. This imputation is not central to the balls-and-bins framework itself. Indeed, BCH perfectly exemplify how to use the balls-and-bins model with shipment-level data when they show that single-country and single-product exporters are more frequent in Chilean data than expected. Given the right data, imputation is no longer needed.

The authors themselves admit that there is no connection between the variation in shipment sizes and their critique of the balls-and-bins model, stating that "whether one utilizes the actual shipment data or some other assumption on shipment size, the resulting balls-and-bins model is not a purely statistical benchmark for evaluating trade zeros in the data" (BCH, p. TK). Their verdict seems to be that, with or without an imputation for shipment size, there is too much economics in the balls-and-bins model for it to serve as a statistical tool.

\footnotetext{
${ }^{2}$ Such a model would need to be evaluated quantitatively. The balls-and-bins model still predicts a sizable presence of single-product, single-destination exporters_-indeed, half or more of those observed in the data.
} 
In AK, we explicitly state that the balls-and-bins model incorporates economic fundamentals in the construction of the bin sizes and number of balls (pp. 2128-2129). It is only the assignment of balls to bins - that is, of shipments to categories - that is random. Indeed, we have devoted ample space to discuss the key features in the calibration of bin sizes and number of balls responsible for the results-highlighting, for example, the importance in the skewness in both dimensions (pp. 2129, 2140, 2144). These key economic features are pervasive across trade models-even those without an explicit treatment of the extensive margin. This is why we concluded that whenever the balls-and-bins model matches a statistic, such statistic is "consistent with a surprisingly large class of trade models.",3

$\mathrm{BCH}$ invite a comparison with the work of Ellison and Glaeser (1997), which, they argue, is devoid of any economic content; unlike the balls-and-bins model. This argument reflects a serious misunderstanding of Ellison and Glaeser (1997). The number of "darts" in that model is the number of establishments in a given industry. Surely this number is the result of economizing behavior - as is the number of shipments. This does not prevent Ellison and Glaeser (1997) to test the hypothesis that the observed concentration establishments can be matched by a random location model, and does not prevent Armenter and Koren (2014) from testing the hypothesis that the frequency of zeros in trade can be matched by a random assignment model.

We interpret the results from $\mathrm{BCH}$ for Chile as indicating that a trade model with a careful treatment of shipping decisions has the potential to outperform existing theories of the extensive margin - as spotted by contrasting the balls-and-bins predictions against the data. There is indeed a growing body of work seeking to understand the economic trade-offs involved in the shipping decisions of exporters: see, for example, Alessandria, Kaboski, and Midrigan (2010); Kropf and Sauré (2014); and Hornok and Koren (2015). We agree that structural modeling of exporters' decisions are helpful and encourage further work in this area. We also reassert that the balls-and-bins benchmark can help researchers to focus on those data statistics that are most useful to distinguish among structural models of trade.

\section{REFERENCES}

Alessandria, George, Joseph P. Kaboski, and Virgiliu Midrigan. 2010. "Inventories, Lumpy Trade, and Large Devaluations.” American Economic Review 100 (5): 2304-39.

-Armenter, Roc, and Miklós Koren. 2014. "A Balls-and-Bins Model of Trade." American Economic Review 104 (7): 2127-51.

Blum, Bernardo S., Sebastian Claro, and Ignatius J. Horstmann. 2016. "A Balls-and-Bins Model of Trade: Comment." American Economic Review 106 (3): 843-51.

Ellison, Glenn, and Edward L. Glaeser. 1997. "Geographic Concentration in U.S. Manufacturing Industries: A Dartboard Approach.” Journal of Political Economy 105 (5): 889-927.

Hornok, Cecília, and Miklós Koren. 2015. "Administrative Barriers to Trade.” Journal of International Economics 96 (S1): S110-S122.

Kropf, Andreas, and Philip Sauré. 2014. "Fixed Costs per Shipment." Journal of International Economics $92(1)$ : 166-84.

\footnotetext{
${ }^{3}$ AK (p. 2127). See also Section V, which formalizes the argument.
} 